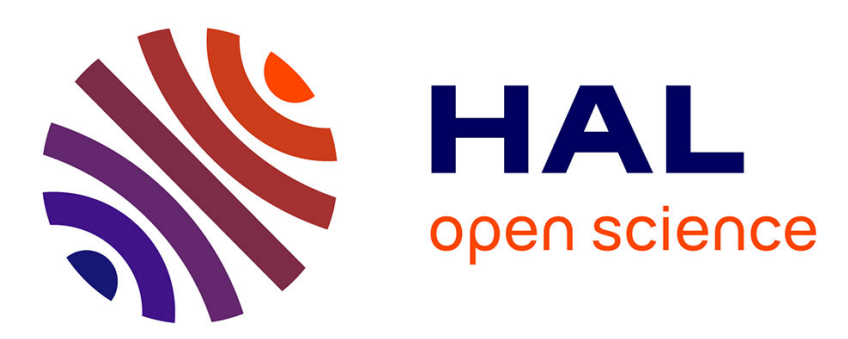

\title{
Testing the intrinsic noise of a coilmagnet actuator for cryogenic gravitational wave interferometers Paolo Falferi
}

\section{To cite this version:}

Paolo Falferi. Testing the intrinsic noise of a coilmagnet actuator for cryogenic gravitational wave interferometers. Classical and Quantum Gravity, 2011, 28 (14), pp.145005. 10.1088/02649381/28/14/145005. hal-00719837

\section{HAL Id: hal-00719837 \\ https://hal.science/hal-00719837}

Submitted on 21 Jul 2012

HAL is a multi-disciplinary open access archive for the deposit and dissemination of scientific research documents, whether they are published or not. The documents may come from teaching and research institutions in France or abroad, or from public or private research centers.
L'archive ouverte pluridisciplinaire HAL, est destinée au dépôt et à la diffusion de documents scientifiques de niveau recherche, publiés ou non, émanant des établissements d'enseignement et de recherche français ou étrangers, des laboratoires publics ou privés. 


\title{
Testing the intrinsic noise of a coil-magnet actuator for cryogenic gravitational wave interferometers
}

\author{
Paolo Falferi \\ Istituto di Fotonica e Nanotecnologie, CNR-Fondazione Bruno Kessler, 38123 Povo, Trento, Italy and INFN, \\ Gruppo Collegato di Trento, Sezione di Padova, 38123 Povo, Trento, Italy \\ e-mail: falferi@ science.unitn.it
}

\begin{abstract}
The third generation gravitational wave (GW) interferometers that will operate underground and at cryogenic temperatures will need a complex and sophisticated control system to satisfy the requirements on the alignment and position of its optics and keep the detector at its working point. The force actuators of the control systems of the present interferometers are for the most part coil-magnet actuators. To check the possibility of using these actuators also at low temperature we have tested the magnetization and the magnetization noise of a SmCo magnet at $4.2 \mathrm{~K}$. The magnetization loss, measured with a fluxgate magnetometer, is 7\%. The magnetization noise has been measured with a SQUID (Superconducting Quantum Interference Device) magnetometer. The application of dc and ac $(0.1 \mathrm{~Hz})$ magnetic fields of amplitude comparable to that needed to produce on the magnet a force large enough for the control system, does not change the measured noise. The equivalent maximum force noise produced by the actuator as a result of the magnetization noise of the magnet has been evaluated. Its effect on the sensitivity of a third generation interferometer (Einstein Telescope) is negligible with respect to the most relevant fundamental noise contributions.
\end{abstract}

PACS numbers: 04.80.Nn, 95.55.Ym, 05.40.Ca, 75.30.Mb, 75.50.Ww

\section{Introduction}

The development of a third-generation ground-based GW observatory, with a target sensitivity of a factor of 100 improved over present detectors, is currently under design study within the framework of the Einstein Telescope (ET) project [1]. Since the first detection of a GW signal is expected to occur in the advanced interferometers [2], currently under construction, the goal of ET, operating in a complementary way with LISA [3], is to open the era of routine GW astronomy.

The most accurate sensitivity model of the Einstein Telescope is referred to as ET-D [4], a xylophonedesign, in which one GW detector is comprised of two individual interferometers: A low-power, cryogenic low-frequency interferometer and a high-power, room-temperature high frequency interferometer. As the present interferometers, ET-D LF (the cryogenic low-frequency interferometer) will require a sophisticated control strategy [5][6] to maintain the proper operating point by means of a low noise actuation system.

At present, the actuation systems used in these detectors are of two kinds: coil-magnet [7] and electrostatic [8]. The electrostatic actuator is currently used in the GEO 600 interferometer and is planned for advanced LIGO. The coil-magnet actuator has been well tested and successfully used at room temperature in the large first-generation interferometers like Virgo and LIGO. This type of actuator is also used in the cryogenic underground prototype detector CLIO [9] but up to now the expected performance has been demonstrated only at room temperature [10]. On the other hand, in some circumstances doubts have been expressed about the reliability of these actuators. In particular there is evidence that the Barkhausen noise produced by the magnets of some actuators could significantly contribute to limit the detector sensitivity [10] [11] [12]. 
Consequently, in view of the use of this actuator in the cryogenic ET D-LF (the model assumes the mirrors at $10 \mathrm{~K}$ and the penultimate suspension mass at $2 \mathrm{~K}$ ), it seems advisable to evaluate the magnetic properties (magnetization and magnetic noise) of the permanent magnets at low temperatures, in particular of the widely used SmCo. These, in fact, could change at low temperature or be inadequate for the expected ET sensitivity.

In general there are many possible problems associated with the coil-magnet actuator. For example the effect of the magnets on the mechanical quality factor of the mirror [13], the parasitic coupling with external magnetic fields [8] [14], or the stress due to the differential thermal contraction between magnets and mirror. In this work we consider only the magnetization change of the magnet due to the cooling from room temperature to $4.2 \mathrm{~K}$ and the magnetic noise produced by the magnet at low temperature which is converted in force noise in the actuator. In particular, possible mechanisms that could occur at low temperature and limit the ET sensitivity are: An excessive loss of magnetization [15], Barkhausen noise triggered by the dc or low frequency signal of the actuator coil [16] [17], thermal magnetic noise [18].

\section{Experimental apparatus}

Measurements have been carried out on a cylindrical SmCo magnet [19], diameter $\approx 10 \mathrm{~mm}$, height $\approx 4 \mathrm{~mm}$, axially magnetized, of the same type used in the Virgo marionette. The magnetic field at room temperature at the top surface of the magnet is $0.36 \mathrm{~T}$. This value has been extrapolated from measurements performed with a fluxgate magnetometer at different distances along the magnet axis and is in good agreement with the nominal value of $\mathrm{B}_{\mathrm{r}}=1 \mathrm{~T}$ given by the manufacturer. For convenience, all the low temperature measurements have been realized at $4.2 \mathrm{~K}$, in liquid helium at atmospheric pressure. The measurements of magnetization change have been performed with a fluxgate magnetometer with cryogenic probe [20] and the magnetic noise measurements with a commercial SQUID system (sensor and electronics) [21].

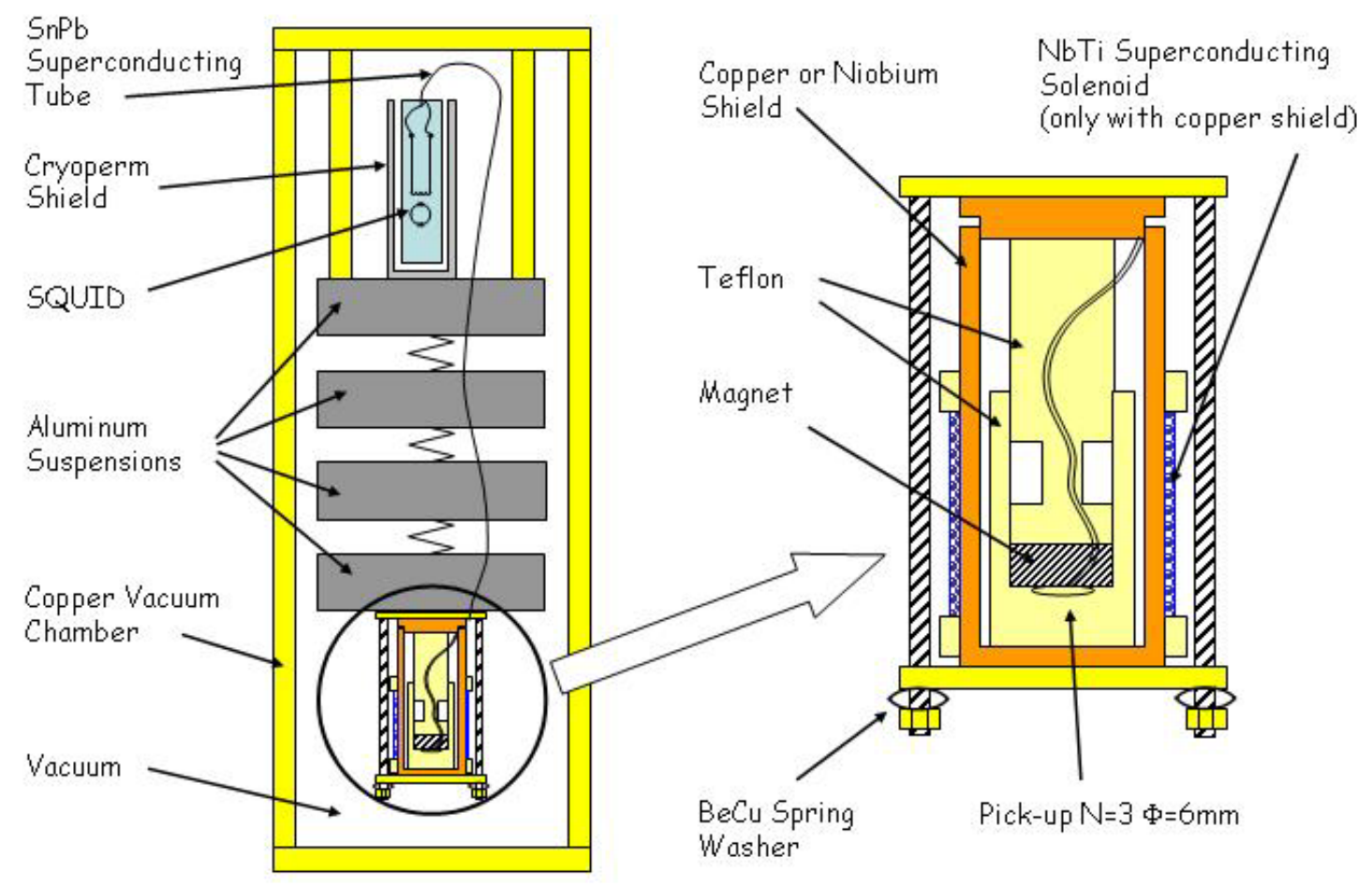

Figure 1. Schematic of the experimental apparatus for the magnetic noise measurements with a detailed view of the shield of the magnet. 
A pick-up (superconducting $\mathrm{NbTi}$ wire, 3 turns, diameter $\approx 6 \mathrm{~mm}, \mathrm{~L}_{\mathrm{p}} \approx 0.16 \mu \mathrm{H}$ ), fixed coaxially on the magnet, is connected (see figure 1) to the input coil of the SQUID sensor $\left(\mathrm{L}_{\mathrm{i}} \approx 1.6 \mu \mathrm{H}\right)$. The current in the input coil causes a flux to be coupled to the SQUID loop through the mutual inductance $\mathrm{M}_{\mathrm{SQ}} \approx 10 \mathrm{nH}$. The magnet and pick-up are contained in a high purity niobium shield (RRR>200) [22] or in a copper shield. The $\mathrm{BeCu}$ spring washers provide a rigid assembly of magnet and pick-up in the Teflon holder without excessive mechanical stress. A superconducting NbTi solenoid is wound on the copper shield in order to apply a dc or low frequency field (the copper shield cut-off frequency is about $10 \mathrm{~Hz}$ ). Although magnet, pick-up and shield are rigidly connected to reduce, as much as possible and at least at low frequencies, relative displacements, it has been necessary to operate in vacuum and with suspensions to reduce the effect of the seismic and acoustic disturbances. The three stage suspensions of the cantilever-mass type [23] were manufactured by electric discharge machining from a single piece of $\mathrm{Al} \mathrm{7075.} \mathrm{They} \mathrm{are} \mathrm{effective} \mathrm{in} \mathrm{the}$ frequency range 100-800 Hz with an expected maximum vertical attenuation of $108 \mathrm{~dB}$ at $282 \mathrm{~Hz}$. Given the limited space available, it was not possible to build suspensions effective also at lower frequencies. The copper vacuum chamber is immersed in liquid helium in a Dewar with Mu-metal shield. A small amount of helium gas (a few mbar before the cooling) is used for the thermalization of the internal assembly and then pumped out to about $10^{-5}$ mbar.

\section{Results and implications for ET}

As regards the magnetization change upon cooling from room temperature to $4.2 \mathrm{~K}$, a reproducible decrease of $7 \%$, that does not represent a problem for the actuator, was observed.

The noise measurements are realized starting from about 2 hours after the cooling in order to permit a sufficient decrease of the signal drift and the SQUID operation.

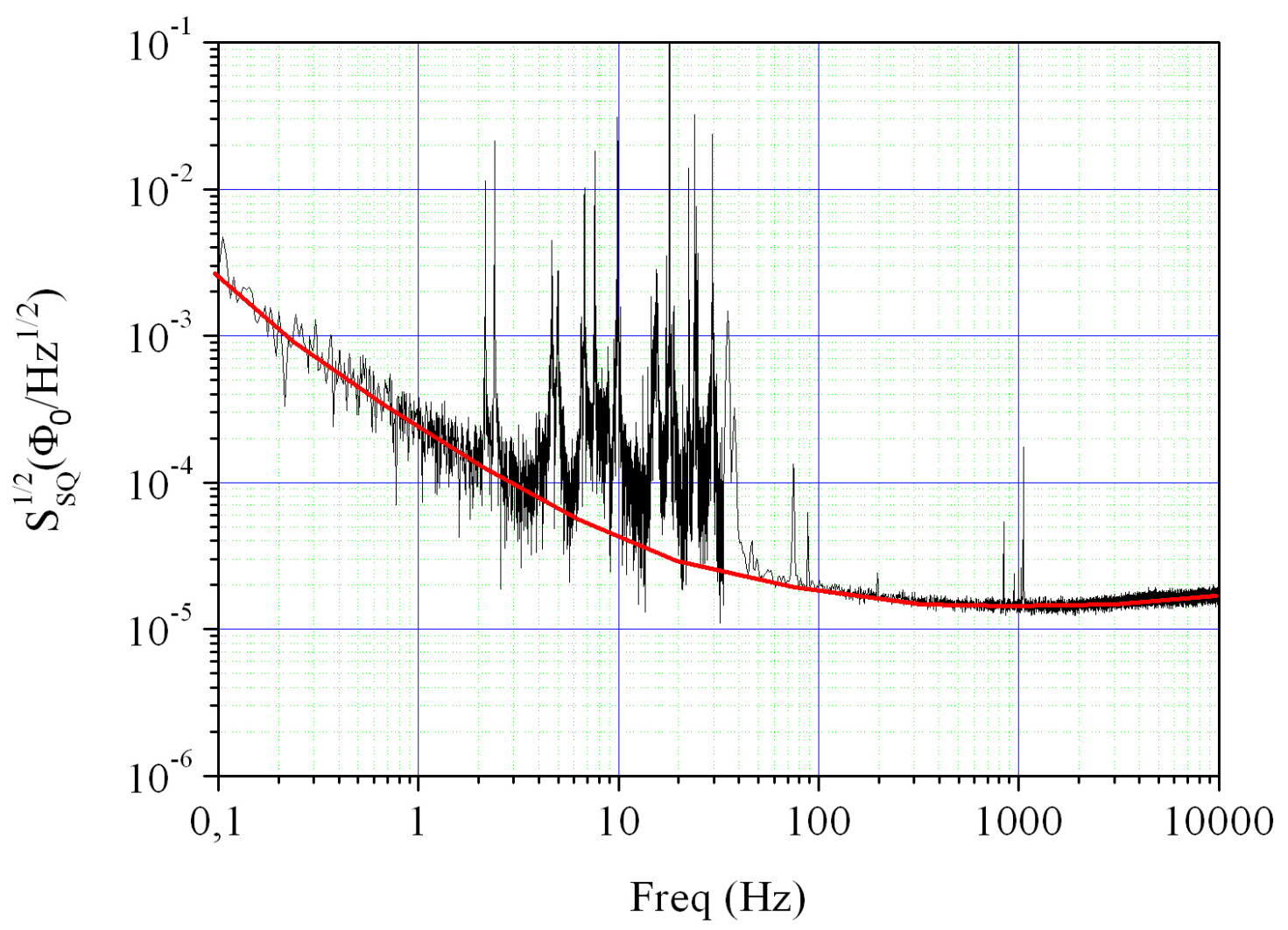

Figure 2. A typical flux noise spectrum at the SQUID loop produced by the magnet in the niobium shield.

Figure 2 shows a typical flux noise spectrum (calculated at the SQUID loop and expressed in units of $\Phi_{0} / \sqrt{ } \mathrm{Hz}$, where $\left.\Phi_{0}=2.07 \times 10^{-15} \mathrm{~Wb}\right)$ produced by the magnet in the niobium shield and obtained by combining two spectra taken with different frequency range and averages. The general characteristics of this 
noise spectrum correspond to the expectations. At the lower frequencies the assembly composed of the magnet, pick-up and niobium shield behaves as a rigid body and the relative displacements between these components are negligible. As a consequence the typical vibrational peaks are not present. On the contrary, these are present at intermediate frequencies. At higher frequencies $(100-1000 \mathrm{~Hz})$ the vibrational noise is filtered by the suspensions.

Several noise measurements have been realized in different configurations: with niobium shield, with copper shield, with the SmCo magnet, with a Teflon cylinder with the same dimensions of the magnet (a reference measurement with a non-magnetic non-conducting material), with a stainless steel cylinder with the same dimensions of the magnet (a non-magnetic conducting material with resistivity similar to that of SmCo). The analysis of these measurements has suggested the following interpretation of the noise spectrum of figure 2. The intrinsic noise of the SQUID, typically around 4-5 $\mu \Phi_{0} / \sqrt{\mathrm{Hz}}$, is negligible. At frequencies higher than $200-300 \mathrm{~Hz}$ the noise is dominated by thermal magnetic noise that is magnetic field fluctuations arising from the thermally agitated motion of electric charges of the conductor (SmCo in this case). This noise can be related to the temperature of the metal via a Nyquist's type relation [24]. A similar noise level has been obtained with the stainless steel cylinder. Vibrational peaks are present between 2 and $100 \mathrm{~Hz}$ (as expected suspensions are effective between 100 and $1000 \mathrm{~Hz}$ ). Between 0.1 and $4 \mathrm{~Hz}$ the noise, of the 1/f-type, is due to the magnet and tends to decrease with time (the noise spectrum shown in figure 2 has been taken about 5 hours after the cooling).

We have also measured the noise produced by the magnet when it is subjected to a dc or low frequency $(0.1$ $\mathrm{Hz}$ ) magnetic field with magnitude comparable to that needed to produce a force of the order of $1 \mathrm{mN}$. These tests have been carried out with the copper shield because, contrary to the niobium shield, it permits us to apply a field generated by a superconducting coil wound on the external surface of the shield (see figure 1). All the tests have shown that the application of magnetic field does not change the noise of the magnet. This result may appear surprising but one has to consider that the magnetic field applied by the coils to maintain the interferometer at the proper operating point is orders of magnitude less than the coercive field of the magnets. It is therefore reasonable to suppose that the Barkhausen noise, if any, is much less than that usually measured at room temperature and at magnetic field values where the slope of the hysteresis curve (the incremental permeability) is much higher.

From the comparison of the noise spectra taken with the niobium shield and the copper shield it is possible to point out some differences due to the shields. The copper shield adds its thermal magnetic noise (low-passlike with cut-off frequency around $10 \mathrm{~Hz}$ ) making the spectra taken in this configuration more noisy (a factor 3 at most), in the range 40-1000 Hz, than those taken with the niobium shield. The spectra taken with the niobium shield show a considerable excess noise below $0.1 \mathrm{~Hz}$ (up to 3 orders of magnitude) probably due to flux creep in niobium.

The frequency range $2-100 \mathrm{~Hz}$ is dominated by vibrational peaks but the shape of the magnet intrinsic noise can be easily guessed and is described by the red curve in figure 2 that we consider as reference noise level for the following calculation of the force noise acting on the mirror.

In general, the force between the coil and the magnet can be expressed as $F=\alpha \mu I$ where $\mu$ is the magnetic moment of the magnet, $I$ the current in the coil and $\alpha$ depends on geometrical factors [7]. As the magnetic field produced by a magnet is proportional to its magnetic moment, the force $\mathrm{F}$ is also proportional to the magnetic field measured at the surface of the magnet (from our measurements, $0.36 \mathrm{~T}$ at room temperature) and hence proportional to the flux picked-up by the pick-up coil connected to the SQUID. We have then

$$
S_{\Phi}^{1 / 2} / \Phi=S_{F}^{1 / 2} / F
$$

where $S_{\Phi}^{1 / 2}$ is the flux noise at the pick-up in $\mathrm{Wb} / \sqrt{\mathrm{Hz}}, \Phi$ is the dc flux at the pick-up in $\mathrm{Wb}, S_{F}^{1 / 2}$ is the force noise of the actuator in $\mathrm{N} / \sqrt{\mathrm{Hz}}$ when the maximum force is generated and $F$ is the maximum force of the actuator in $\mathrm{N}$. Here we are interested in the effect of the magnetization noise. For this reason the current noise in the coil, although important in the practical actuators [25], has been neglected.

The noise spectrum of figure 2 is expressed in $\Phi_{0} / \sqrt{ } \mathrm{Hz}$ and refers to the noise $S_{S Q}{ }^{1 / 2}$ measured at the SQUID loop. It can be shown (see for example [26]) that the noise referred to the pick-up at the magnet surface in $\mathrm{Wb} / \sqrt{\mathrm{Hz}}$ is given by

$$
S_{\Phi}^{1 / 2}(\mathrm{~Wb} / \sqrt{H z})=\left(2.07 \times 10^{-15}\left(L_{p}+L_{i}\right) / M_{S Q}\right) S_{S Q}^{1 / 2}\left(\Phi_{0} / \sqrt{H z}\right) \approx 3.3 \times 10^{-13} S_{S Q}^{1 / 2}\left(\Phi_{0} / \sqrt{H z}\right)
$$


The estimate of the dc flux that crosses the pick-up is $\Phi=B \beta_{4.2} N S$ where $B=0.36 \mathrm{~T}$ is the extrapolated magnetic field at the magnet surface at room temperature, $\beta_{4.2}=(1-7 \%)=0.93$ is the factor that represents the magnetization reduction at $4.2 \mathrm{~K}, N=3$ the pick-up number of turns and $S=\pi\left(3 \times 10^{-3}\right)^{2} \mathrm{~mm}^{2}$ the pick-up area. From these values $\Phi \approx 2.8 \times 10^{-5} \mathrm{~Wb}$. It is worthwhile to stress that the ratio $\Phi / S_{\Phi}^{1 / 2}$ that can be considered the "dynamic range" of the magnet is about $10^{12}$, a value orders of magnitude higher than that of the drivers used to control the current in the actuator coil.

From the spectrum of figure 2 and the values of $S_{\Phi}^{1 / 2}, \Phi$ and the maximum force needed one can calculate the force noise from equation (1). For example, for $F=1 \mathrm{mN}$ the force noise at $10 \mathrm{~Hz}$ is about $5.3 \times 10^{-16}$ $\mathrm{N} / \sqrt{\mathrm{Hz}}$. A rough estimate of the strain noise contribution for the ET-D LF model due to an actuator based on the considered magnet, can be calculated as follows

$S_{h}^{1 / 2} a c t=G(v) S_{F}^{1 / 2}=G(v) F S_{\Phi}^{1 / 2} / \Phi$

where $v$ is the frequency and $G(v)$ is the transfer function that converts the force noise in the equivalent strain noise and in general depends on the interferometer arm, the point where the actuator force is applied (for example the mirror test mass) and the particular seismic isolation system.

If we consider the actuator that operates between mirror and reaction mass and represent the mirror as a free test mass then

$$
G(v)=1 / 4 \pi^{2} m v^{2} L
$$

where $m$ is the mirror mass and $\mathrm{L}$ the interferometer arm length. A conservative estimate of the maximum force $\mathrm{F}$ requested to control the mirror of the ET-D LF can be obtained by scaling the force requested for Virgo $(10 \mu \mathrm{N}$ [7]) with the mirror mass ratio (20 kg for Virgo, $200 \mathrm{~kg}$ for ET-D LF [4]). For a maximum force $F=100 \mu \mathrm{N}$ and an arm length of $10 \mathrm{~km}$, the equivalent strain noise spectrum due to the magnet of the mirror - reaction mass actuator is shown in figure 3 (red dashed line) together with the ET-D LF total noise (black solid line).

If, as in Virgo, the ET-D LF suspension control will be realized in a hierarchical way with actuators also on the last stage of the suspensions (the so-called marionetta), we can estimate the effect of the magnet noise of these actuators in the following way. We consider the marionetta as a free mass $M=400 \mathrm{~kg}$ and the resonance frequency of the mirror pendulum $\left(v_{0}=0.3 \mathrm{~Hz}\right)$ much less than the considered noise frequency range. In this case

$$
G(v)=v_{0}^{2} / 4 \pi^{2} M v^{4} L
$$

Assuming for the marionette control a maximum force of $5 \mathrm{mN}$ then the equivalent strain noise spectrum due to the magnet of the marionette actuator is shown in figure 3 (green dotted line).

From these rough but conservative estimations we can conclude that the strain noise contributions at the mirror level, induced by the coil-magnet actuators of the marionette and mirror, are negligible. 


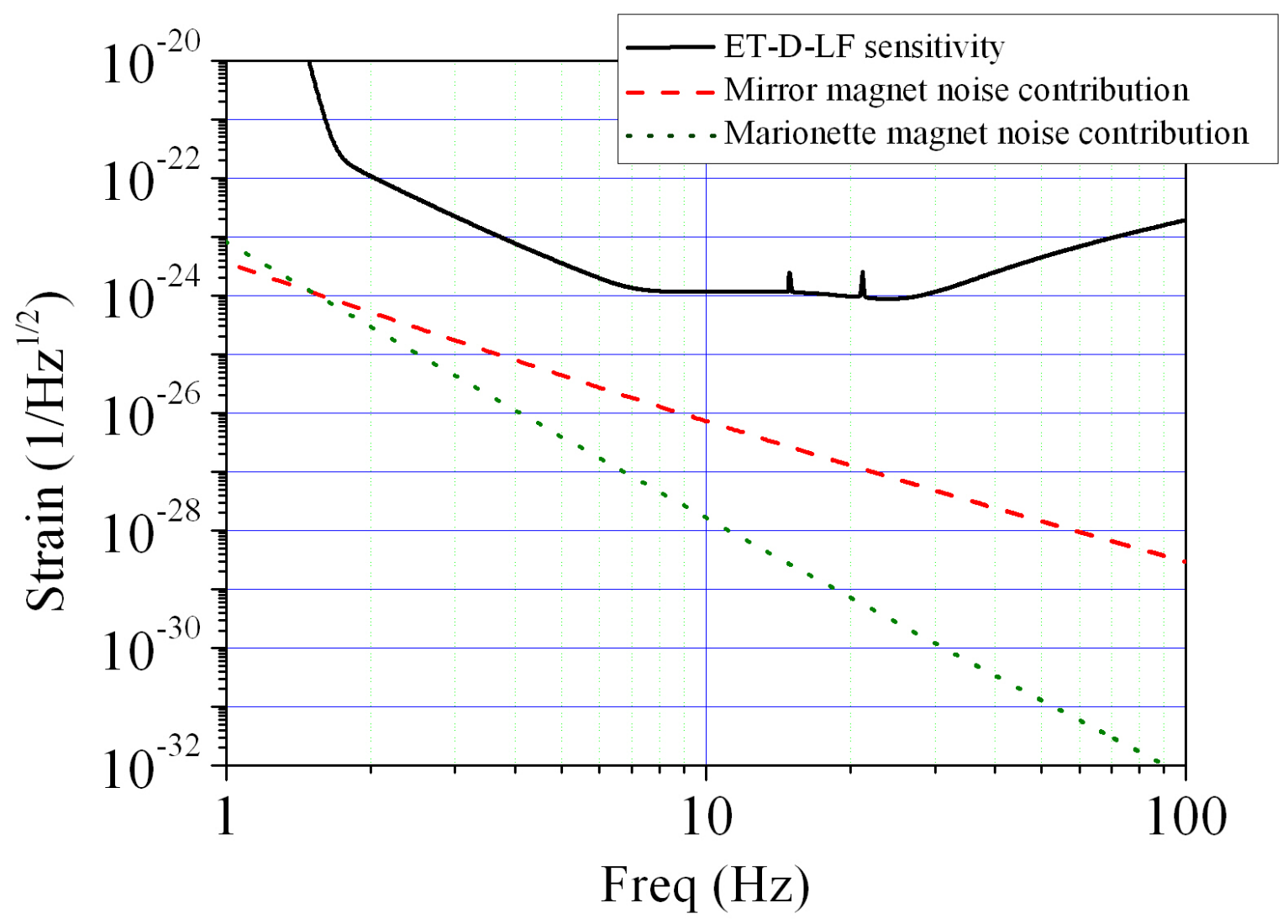

Figure 3. Equivalent strain noise spectrum due to the magnet of the mirror - reaction mass actuator (red dashed line) and to the magnet of the marionette actuator (green dotted line) in comparison with the ET-D LF total noise (black solid line). The noise contributions of the actuators have been calculated assuming for the marionette control a maximum force of $5 \mathrm{mN}$ and for the mirror control a maximum force of $100 \mu \mathrm{N}$.

\section{Further developments}

In the ET-D LF model [4], the Brownian thermal noise of the mirror substrate (silicon) is negligible compared to other fundamental noises. This result holds under the assumption that the value of the order of $10^{-9}$ for the mechanical loss of silicon at $10 \mathrm{~K}$ reported in [27] remains unchanged even in operating conditions. Unfortunately, as mentioned in the introduction, there are experimental results indicating that the control actuators, both coil-magnet [13] and electrostatic [28], could add losses much higher than $10^{-9}$.

An interesting alternative for the cryogenic interferometers is the actuator proposed in [29] in which a repulsive force is exerted by a reaction mass coil on a superconducting film coated on the mirror. The expected benefit is the low mechanical loss added by the superconducting film that can be realized with a very small amount of material. The major drawback is that the actuator works only at low temperature (below the critical temperature of the superconducting film). As a consequence, the long cooling time of the payload could prevent a rapid development and debugging of the control system.

As is described in [29] this actuator can generate enough force for the control of a cryogenic interferometer like LCGT, the next Japanese GW telescope project [30], but from the point of view of the intrinsic noise, for example the noise due to the flux creep, experimental results are still lacking. The experimental apparatus described in this paper can be used to fill the gap by repeating on the superconducting film the measurements performed on the SmCo magnet.

\section{Acknowledgments}

The author thanks Pierino Gennara and Enzo Bissiato for their skilled technical assistance and Antonio Pontin, Gabriele Silvestrin and Jean-Pierre Zendri for suggestions and support in the design and fabrication of the mechanical suspensions. 


\section{References}

[1] http://www.et-gw.eu/

[2] Sathyaprakash B S and Schutz B F 2009 Physics, astrophysics and cosmology with gravitational waves Living Rev. Rel. 122

[3] Shaddock D A 2008 Space-based gravitational wave detection with LISA Class. Quantum Grav. 25, 114012

[4] Hild S et al. 2011 Sensitivity Studies for third Generation Gravitational Wave Observatories Class. Quantum Grav. 28, 094013

[5] Fritschel P et al. 1998 Alignment of an interferometric gravitational wave detector Appl. Opt. 3728

[6] Acernese F et al. 2008 The real-time distributed control of the Virgo interferometric detector of gravitational waves IEEE Trans. Nucl. Sci. 55302

[7] Bernardini M, Majorana E, Puppo P, Rapagnani P, Ricci F and Testi G 1999 Suspension last stages for the mirrors of the Virgo interferometric gravitational wave antenna Rev. Sci. Instrum. 703453

[8] Grote H (for the LIGO Scientific Collaboration) 2008 The Status of GEO 600 Class. Quantum Grav. 25 114043

[9] Miyoki S et al. 2006 The CLIO project Class. Quantum Grav. 23 S231

[10] Agatsuma K et al. 2010 Thermal-noise-limited underground interferometer CLIO Class. Quantum Grav. 27084022

[11] Smith J R et al. 2009 The path to the enhanced and advanced LIGO gravitational-wave detectors Class. Quantum Grav. 26114013

[12] Abbott B P et al. 2009 LIGO: the Laser Interferometer Gravitational-Wave Observatory Rep. Prog. Phys. 72076901

[13] Amico P, Bosi L, Carbone L, Gammaitoni L, Marchesoni F, Punturo M, Travasso F and Vocca H 2002 Mechanical quality factor of large mirror substrates for gravitational wave detectors Rev. Sci. Instrum. $\mathbf{7 3}$ 179

[14] Accadia T et al. 2010 Status and perspectives of the Virgo gravitational wave detector Journal of Physics: Conference Series 203012074

[15] Poggiani R 2009 Materials and components of possible interest for cryogenic operation of Einstein Telescope ET Technical Note ET-026-09 available at https://tds.ego-gw.it/itf/tds/index.php

[16] Basso V, Beatrice C, Bertotti G, Durin G, Lo Bue M, Sasso C P 2004 Barkhausen noise in nucleationtype hard magnetic materials Journal of Magnetism and Magnetic Materials 272-276 e539

[17] Diao Z, Nowak E R, Feng G, and Coey J M D 2010 Magnetic noise in structured hard magnets Phys. Rev. Lett. 104047202

[18] Varpula $\mathrm{T}$ and Poutanen T 1984 Magnetic field fluctuations arising from thermal motion of electric charge in conductors J. Appl. Phys. 554015

[19] Alga Magneti www.algamagneti.com

[20] Mag-01H unit with Mag Probe F, Bartington Instruments, www.bartington.com

[21] Model 50 dc SQUID sensor and Model 550 Controller, Quantum Design, www.qdusa.com

[22] Tokyo Denkai Co., Ltd, 3-20 Higashisuna 1-chome, Koto-ku, Tokyo 136-0074, Japan

[23] Veitch P J 1991 Vibration isolation of distributed mechanical oscillators by mechanical suspensions with application to resonant-mass gravitational radiation antennae Rev. Sci. Instrum. 62140

[24] Beyer J, Drung D, Kirste A, Engert J, Netsch A, Fleischmann A, and Enss C 2007 A magnetic-fieldfluctuation thermometer for the $\mathrm{mK}$ range based on SQUID-magnetometry IEEE. Trans. Appl. Supercond. 17760

[25] Gennai A 2009 DAC noise effect on VIRGO sensitivity Virgo Technical Note VIR-006A-09 available at https://tds.ego-gw.it/itf/tds/index.php

[26] Clarke J and Braginski A 2004 The SQUID Handbook vol I ed J Clarke and A I Braginski (Weinheim: Wiley-VCH) p 19

[27] McGuigan D F, Lam C C, Gram R Q, Hoffman A W, Douglass D H and Gutche H W 1978 Measurements of the mechanical Q of single-crystal silicon at low temperature J. Low Temp Phys $\mathbf{3 0} 621$

[28] Mitrofanov V P, Styazhkina N A and Tokmakov K V 2002 Test mass damping associated with electrostatic actuator Class. Quantum Grav. 192039

[29] Sato N et al. 2003 Force measurements of a superconducting-film actuator for a cryogenic interferometric gravitational-wave detector Cryogenics $\mathbf{4 3} 425$

[30] Kuroda K (on behalf of the LCGT Collaboration) 2010 Status of LCGT Class. Quantum Grav. 27 084004 